\title{
Influência da Alocação de Pólos Associados a Curvas Algébricas com $g=1$ na Determinação de Grupos Fuchsianos
}

\author{
Erika Patricia Dantas de Oliveira Guazzi ${ }^{1}$ \\ UTFPR, Campo Mourão, PR \\ Reginaldo Palazzo Júnior ${ }^{2}$ \\ FEEC, UNICAMP, Campinas, SP
}

\begin{abstract}
Resumo. O presente trabalho aborda a maneira pela qual a alocação das singularidades de uma curva algébrica, inicialmente em $\Delta$ (ou seja, os pólos de uma equação diferencial fuchsiana) e por isometria transferidas para $\mathbb{H}^{2}$, influencia na determinação do grupo fuchsiano associado e, consequentemente, no recobrimento da superfície. E mais, se uma relação de similaridade entre curvas algébricas corresponde a um isomorfismo entre os respectivos grupos fuchsianos.
\end{abstract}

Palavras-chave. Pólos, Grupo Fuchsiano, Gênero 1, Curvas Algébricas, Isometria

\section{Introdução}

No presente estudo, utilizam-se dois modelos dentre os quatro modelos que caracterizam a geometria hiperbólica, $[1,4]$, a saber, os modelos do semi-plano superior, $\mathbb{H}^{2}=\{z \in \mathbb{C}: \operatorname{Im}(z)>0\}$, e do disco aberto unitário, $\Delta=\left\{(x, y) \in \mathbb{R}^{2}: x^{2}+y^{2}<1\right\}$. Estes modelos são isométricos a partir da isometria dada por

$$
F(z)=\frac{(1+z) i}{1-z}
$$

Essa isometria permite a correspondência entre cada singularidade da curva algébrica em $\Delta$ e em $\mathbb{H}^{2}$.

Dadas tais premissas, o presente trabalho aborda a maneira pela qual a alocação das singularidades de uma curva algébrica, inicialmente em $\Delta$ (ou seja, os pólos de uma equação diferencial fuchsiana) e por isometria transferidas para $\mathbb{H}^{2}$, influencia na determinação do grupo fuchsiano associado e, consequentemente, no recobrimento da superfície. E mais, se uma relação de similaridade entre curvas algébricas corresponde a um isomorfismo entre os respectivos grupos fuchsianos.

Este trabalho está organizado da seguinte maneira. Na Seção 2, serão apresentadas as equações diferenciais fuchsianas associadas a três curvas algébricas de gênero 1 e grau

\footnotetext{
${ }^{1}$ erikapatricia@utfpr.edu.br

${ }^{2}$ palazzo@dt.fee.unicamp.br
} 
4. Em particular, as singularidades de tais polinômios estão contidas no interior ou na fronteira da circunferência de centro $(0,0)$ e raio 1, ou seja, $\Delta$ ou $\partial \Delta=\left\{(x, y) \in \mathbb{R}^{2}\right.$ tal que $\left.x^{2}+y^{2}=1\right\}$, [5]. Na Seção 3, apresenta-se um algoritmo para a obtenção dos geradores dos grupos fuchsianos tendo as raízes (singularidades) do polinômio como vértices de um polígono do qual serão obtidos os grupos fuchsianos associados. Para isso, o procedimento proposto por Whittaker, [6], será utilizado. Na Seção 4 são apresentados alguns exemplos da obtenção do respectivo grupo fuchsiano, com os respectivos detalhamentos. Em particular, foram consideradas curvas algébricas trabalhadas na Seção 2. Na Seção 5, foi realizada a comparação entre os grupos fuchsianos obtidos nos exemplos da Seção 4, cujas curvas algébricas tem o mesmo grau, especialmente se é possível ou não o estabelecimento de um isomorfismo entre os grupos fuchsianos. Por fim, na Seção 6, serão apresentadas as conclusões. Ressalta-se o uso do software Scilab ${ }^{3}$, um software livre, na resolução numérica dos exemplos que serão desenvolvidos neste artigo.

\section{Equação Diferencial Fuchsiana Associada}

Nesta seção, primeiramente são apresentadas as condições que uma equação diferencial deve satisfazer para ser uma equação diferencial fuchsiana. Em seguida, determinam-se as equações diferenciais fuchsianas associadas a curvas algébricas de gênero 1 e grau 4, ou seja, os pólos de tais equações diferencias fuchsianas são as raízes de tais curvas algébricas.

Recorda-se que uma equação diferencial

$$
\ddot{y}(z)+p(z) \dot{y}(z)+q(z) y(z)=0
$$

é dita fuchsiana se os polos de $p(z)$ são de ordem no máximo 1 e os polos de $q(z)$ são no máximo 2. E mais, em [2] apresentam-se condições explícitas para a obtenção de tal equação diferencial dado que se conhecem as singularidades e, consequentemente, a curva algébrica associada, a saber,

$$
p(z)=\frac{A_{1}}{z-z_{1}}+\cdots+\frac{A_{n}}{z-z_{n}}+k_{1}
$$

$\mathrm{e}$

$$
q(z)=\frac{B_{1}}{\left(z-z_{1}\right)^{2}}+\frac{C_{1}}{z-z_{1}} \cdots+\frac{B_{n}}{\left(z-z_{1}\right)^{2}}+\frac{C_{n}}{z-z_{1}}+k_{1}
$$

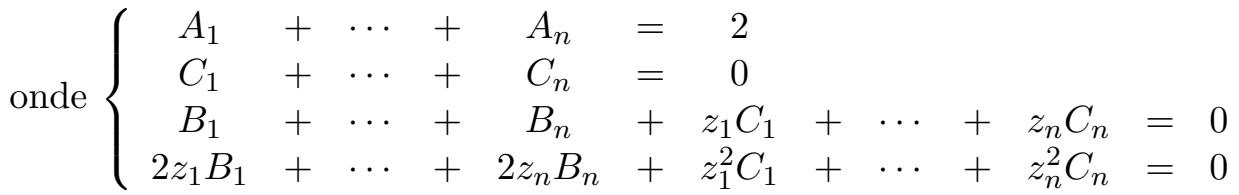

Faz-se uso do software Scilab na resolução de tal sistema, a fim de determinar as expressões $p(z)$ e $q(z)$.

Agora, apresenta-se a obtenção das equações diferenciais fuchsianas associadas às curvas algébricas dadas.

\footnotetext{
${ }^{3}$ http://www.scilab.org/
} 
Exemplo 2.1. Dada a curva algébrica $y^{2}=z^{4}+1$ e seguindo o processo, apresentado acima, para determinar a equação diferencial fuchsiana, obtém-se que

$$
n_{0}(z) y^{\prime \prime}(z)+n_{1}(z) y^{\prime}(z)+n_{2}(z) y(z)=0
$$

é a equação diferencial fuchsiana associada a curva dada, onde

$$
\begin{gathered}
n_{0}(z)=z^{4}+1, \\
n_{1}(z)=2(z+0.71-0.71 i)(z+0.71+0.71 i)(z-0.71+0.71 i)+k_{1}\left(z^{4}+1\right),
\end{gathered}
$$

$e$

$$
n_{2}(z)=k_{2}\left(z^{4}+1\right) .
$$

Exemplo 2.2. Agora, associada a curva algébrica $y^{2}=z^{4}+z^{3}+z^{2}+z+1$ o processo acima fornece

$$
m_{0}(z) y^{\prime \prime}(z)+m_{1}(z) y^{\prime}(z)+m_{2}(z) y(z)=0
$$

é a equação diferencial fuchsiana associada a curva dada, onde

$$
\begin{gathered}
m_{0}(z)=z^{4}+z^{3}+z^{2}+z+1, \\
m_{1}(z)=2(z-0.31-0.95 i)(z+0.81-0.59 i)(z+0.81+0.59 i)+k_{1}\left(z^{4}+z^{3}+z^{2}+z+1\right), \\
m_{2}(z)=k_{2}\left(z^{4}+z^{3}+z^{2}+z+1\right) .
\end{gathered}
$$

Exemplo 2.3. Agora, munido da curva algébrica $y^{2}=5 z^{4}+4 z^{3}+3 z^{2}+2 z+1$, para determinar a equação diferencial fuchsiana associada, faz-se uso do processo apresentado em Barata 2005. Daí, obtem-se que

$$
h_{0}(z) y^{\prime \prime}(z)+h_{1}(z) y^{\prime}(z)+h_{2}(z) y(z)=0
$$

é a equação diferencial fuchsiana associada a curva algébrica dada, onde

$$
h_{0}(z)=5 z^{4}+4 z^{3}+3 z^{2}+2 z+1
$$

$h_{1}(z)=\left[2(z-0.14-0.69 i)(z+0.54-0.36 i)(z-0.14+0.68 i)+k_{1}\left(5 z^{4}+4 z^{3}+3 z^{2}+2 z+1\right)\right]$,

$$
h_{2}(z)=k_{2}\left(5 z^{4}+4 z^{3}+3 z^{2}+2 z+1\right) .
$$

Nota-se que, apesar de tais curvas terem o mesmo número de raízes, implicando em apresentarem o mesmo valor do gênero e mesmo grau, elas conduzem a equações diferenciais fuchsianas diferentes. 


\section{Algoritmo para Obtenção do Grupo Fuchsiano}

Esta seção tem como objetivo apresentar um procedimento para a obtenção dos geradores do grupo fuchsiano, identificando, dessa forma, a região fundamental onde a referida curva algébrica é uniformizada. Os geradores do grupo fuchsiano são obtidos através do quociente de soluções linearmente independentes da equação diferencial linear fuchsiana de segunda ordem. O procedimento em questão tem como base a proposta apresentada por Whittaker, [6]. Equivalentemente, por meio da localização das raízes de uma curva algébrica, raízes essas que podem estar localizadas na fronteira ou no interior do disco de raio unitário, $\Delta$, tem-se como objetivo construir o polígono hiperbólico, identificar o grupo fuchsiano tendo como geradores as transformações elípticas associadas a cada um dos lados do referido polígono, e deste obter o subgrupo fuchsiano tendo como geradores as transformações hiperbólicas (produto das transformações elípticas por uma transformação elíptica fixada). Este subgrupo dará origem à região fundamental da superfície compacta orientável, região esta que uniformizará a curva algébrica.

\section{Algoritmo para Obtenção do Grupo Fuchsiano}

1- Determinar as raízes da curva algébrica em $\Delta$ e em $\mathbb{H}^{2}$;

2- Construir o polígono formado pelas raízes em $\mathbb{H}^{2}$;

3- Para cada lado do polígono hiperbólico, determinar a transformação elíptica, $S_{i}=\frac{a z+b}{c z+d}, \operatorname{com} a d-b c \neq 0$ e $\left|\operatorname{tr}\left(S_{i}\right)\right|<2 ;$

4- Verificar se $S_{i} * S_{i}=I d$;

- Caso $S_{i} * S_{i} \neq I d$, normalizar $S_{i}$ e denote $S_{i}$ normalizado por $N_{i}$;

5- Fixar uma das transformações elípticas, por exemplo $N_{i}$, e calcular os produtos $N_{i} * N_{j}$, para todo $j \neq i$

6- Verificar se as transformações $N_{i} * N_{j}$ são hiperbólicas, isto é, $\left|\operatorname{tr}\left(N_{i} * N_{j}\right)\right|>2$, com $i$ fixo e para todo $j \neq i$

7- Os geradores do grupo fuchsiano são especificados pelo conjunto dos $N_{i} * N_{j}$.

\section{Grupos Fuchsianos Relacionados a Curvas Algébricas de Gênero 1}

Agora, serão apresentados os detalhes da aplicação do algoritmo dado na Seção 3 aos três polinômios desenvolvidos na Seção 2. Ressalta-se que devido a redução de espaço não foi colocado os detalhes para a obtenção das transformações $S_{i}$.

Exemplo 4.1. Considere o polinômio $p_{1}(z)=z^{4}+1$, cujas raízes $z_{1}=-0.71+0.71 i$, $z_{2}=-0.71-0.71 i, z_{3}=0.71+0.71 i$ e $z_{4}=0.71-0.71 i$ estão na fronteira do disco de raio unitário. 
Ao aplicar o Algoritmo para Obtenção do Grupo Fuchsiano, obtem-se os geradores do grupo fuchsiano

$$
G_{1}=\left\langle\frac{2.41 z+3.41}{0.59 z+1.24}, \quad 34.29 z, \quad \frac{2.41 z-3.41}{-0.59 z+1.24}\right\rangle
$$

Exemplo 4.2. Considere o polinômios $p_{2}(z)=z^{4}+z^{3}+z^{2}+z+1$, cujas raízes $z_{1}=0.31+0.95 i, z_{2}=-0.81+0.59 i, z_{3}=-0.81-0.59 i, z_{4}=0.31-0.95 i$ estão sobre a fronteira de $\Delta$. Observa-se que, diferentemente do exemplo acima, tais raízes não formam um polígono regular.

Ao utilizar a isometria entre $\Delta e \mathbb{H}^{2}$ dada pela equação (1), obtem-se para cada singularidade em $\Delta$ a singularidade correspondente em $\mathbb{H}^{2}$. Assim, $F\left(z_{i}\right)=w_{i}, i=1, \ldots, 5$.

Desse modo, as raízes correspondentes acima são tomadas como os vértices do polígono não regular.

Agora, ao seguir os passos do Algoritmo para Obtenção do Grupo Fuchsiano dado na Seção 3, são obtidos os geradores do grupo fuchsiano $G_{2}$, dado por

$$
G_{2}=\left\langle\frac{2.62 z+2.23}{1.18 z+1.38}, \quad 17.79 z, \quad \frac{2.62 z-2.23}{-1.18 z+1.38}\right\rangle
$$

Exemplo 4.3. Considere o polinômio $p(z)=5 z^{4}+4 z^{3}+3 z^{2}+2 z+1$, cujas raízes $z_{1}=0.14+0.68 i, z_{2}=-0.54+0.36 i, z_{3}=-0.54-0.36 i$ e $z_{4}=0.14-0.68 i$ estão no interior de $\Delta$. Observa-se ainda que tais singularidades são os vértices de um polígono não regular.

É possivel, agora, seguir os passos do Algoritmo para Obtenção do Grupo Fuchsiano.

Assim, chega-se aos os geradores do grupo fuchsiano

$$
G_{3}=\left\langle L_{1} L_{2}, L_{1} L_{3}, L_{1} L_{4}\right\rangle
$$

onde

$$
\begin{gathered}
L_{1} L_{2}=\frac{(2.18+0.52 i) z+(1.72+0.38 i)}{(1.17+0.26 i) z+(1.36+0.08 i)} ; \quad L_{1} L_{3}=\frac{3.26 z+0.0004 i}{-0.003 z+0.31} ; \\
L_{1} L_{4}=\frac{(2.18-0.52 i) z+(-1.72+0.38 i)}{(-1.18+0.26 i) z+(1.36-0.08 i)}
\end{gathered}
$$

Chama-se a atenção ao fato do traço dos geradores ser complexo, entretanto o valor absoluto não está entre 0 e 4 .

Em cada exemplo acima, a interação entre os geradores obtidos permite o recobrimento da superfície definida pelo semiplano superior.

\section{Comparação}

Naturalmente surge a indagação se é possível construir um isomorfismo/equivalência também entre os grupos fuchsianos determinados na Seção 4. 
A fim de responder tal questão, primeiramente recorda-se que duas transformações $A$ e $B$ são equivalentes se, e somente se, existe uma transformação $C$ tal que

$$
A=C B C^{-1}
$$

Como cada grupo possui 3 geradores, devemos verificar a existência de uma única transformação $C$ que conduza à equivalência entre os geradores dos grupos em consideração. Por exemplo, dados $G=\left\langle A_{1}, A_{2}\right\rangle$ e $H=\left\langle B_{1}, B_{2}\right\rangle$ dois grupos fuchsianos, deve-se, se possível, apresentar uma transformação $C$ que satisfaz $A_{1}=C B_{1} C^{-1}$ e $A_{2}=C B_{2} C^{-1}$. Verificadas tais relações, é possível afirmar que os grupos $G$ e $H$ são isomorfos.

A seguir realizamos as comparações entre os grupos fuchsianos $G_{1}, G_{2}$ e $G_{3}$ obtidos nos exemplos da Seção 4.

Exemplo 5.1. Considere os grupos fuchsianos $G_{1}$ e $G_{2}$ associados às curvas algébricas $y^{2}=p_{1}(z)$ e $y^{2}=p_{2}(z)$, respectivamente. Tais grupos são equivalentes se $\exists C$ não nula, tal que $A_{i}=C B_{j} C^{-1}$, com $A_{i} \in G_{1}$ e $B_{j} \in G_{2}$.

Assim, iniciamos a procura da transformação $C$ mediante a fixação do gerador $N_{1} N_{2}$. Em seguida, ao supor que existe tal transformação $C$, calculamos, $N_{1} N_{2}=C * M_{1} M_{2} * C^{-1}$, que fornece $C=0$. Desse modo, tal resultado não satisfaz as condições postas. Passamos à próxima hipótese, a saber, $N_{1} N_{2}=C * M_{1} M_{3} * C^{-1}$, que novamente fornece $C=0 . D a$ mesma maneira, $N_{1} N_{2}=C * M_{1} M_{4} * C^{-1}$, cujos cálculos fornecem $C=0$.

Logo, fica evidente a não equivalência de $N_{1} N_{2}$ com qualquer um dos geradores do segundo grupo, $G_{2}$. É possivel concluir, portanto, que não existe um isomorfismo entre os grupos fuchsianos $G_{1}$ e $G_{2}$.

Exemplo 5.2. Considere os grupos fuchsianos $G_{1}$ e $G_{3}$ associados às curvas algébricas $y^{2}=p_{1}(z)$ e $y^{2}=p_{3}(z)$, respectivamente. Ao proceder como no Exemplo 5.1, exibemos a não equivalência de $N_{1} N_{2}$ com qualquer um dos geradores do segundo grupo, $G_{3}$. Logo, temos a não existência de isomorfismo entre os grupos fuchsianos $G_{1}$ e $G_{3}$.

Exemplo 5.3. Considere os grupos fuchsianos $G_{2}$ e $G_{3}$ associados as curvas algébricas $y^{2}=p_{2}(z)$ e $y^{2}=p_{3}(z)$, respectivamente. Tais grupos são equivalentes se $\exists C$ transformação não nula tal que $A_{i}=C B_{j} C^{-1}$, com $A_{i} \in G_{1}$ e $B_{j} \in G_{2}$. Novamente, ao utilizarmos o procedimento anterior obtemos que $M_{1} M_{2}$ não é equivalente a nenhum dos geradores do segundo grupo, $G_{3}$. Consequentemente, segue a não existência de isomorfismo entre os grupos fuchsianos $G_{2}$ e $G_{3}$.

Os exemplos considerados evidenciam a não existência de um isomorfismo entre os grupos fuchsianos obtidos através de curvas algébricas com o mesmo número de raízes, logo com o mesmo grau, portanto com o mesmo gênero. Diante disso, segue o resultado estabelecido na proposição a seguir.

Proposição 5.1. Dadas curvas algébricas de mesmo grau, logo com o mesmo número de raízes e mesmo gênero, os correspondentes grupos fuchsianos derivados não são isomorfos. 


\section{Conclusões}

Diante do exposto, obtevemos, para cada curva algébrica dada, sua respectiva equação diferencial fuchsiana. Tal procedimento gerou equações diferenciais distintas, como verificado na Secão 2. Vale recordar que as transformações de Mobius resultam do quociente das soluções LIs da equação diferencial. E mais, os grupos fuchsianos obtidos para cada curva algébrica são distintos, conforme verificado na Seção 4. Além disso, os grupos fuchsianos associados às curvas algébricas consideradas não são isomorfos, pois dados quaisquer dois dos grupos fuchsianos considerados, e os respectivos geradores, não existe uma transformação de Mobius $C$ tal que satisfaça a equação (2). Portanto, tais fatos convergem paralelamente para a mesma conclusão, a saber: o mesmo número de singularidades não leva a grupos fuchsianos isomorfos.

\section{Agradecimentos}

À UTFPR/CM pela oportunidade de pesquisa em tempo integral. À FAPESP e CNPq pela apoio financeiro durante o período desta pesquisa. Ao Dinter entre UTFPR/CM e UNICAMP que possibilitou a presente pesquisa.

\section{Referências}

[1] J. W. Anderson. Hyperbolic Geometry. In Springer Undergraduate Mathematics Series. Springer, 2008.

[2] J. C. A. Barata. Notas para um Curso de Física-Matemática. São Paulo, Brasil.

[3] G. Kristensson. Second Order Differential Equations: Special Functions and Their Classification. Springer, 2010.

[4] A. J. Oliveira, Uniformização de Curvas Algébricas Associadas a Sequências de Farey Através de Equações Diferenciais Fuchsianas na Proposta de Novos Sistemas de Comunicação, Tese de Doutorado, Unicamp, 2017.

[5] J. A. Pereira, Zeros de Polinômios Auto-Recíprocos Reais no Círculo Unitário, Dissertação de Mestrado, Unesp, 2015.

[6] J. M. Whittaker, The uniformisation of algebraic curves, Journal of the London Mathematical Society., 1930. 